\title{
Aqueous Cytokines as Predictors of Macular Edema in Patients with Diabetes following Uncomplicated Phacoemulsification Cataract Surgery
}

\author{
Ning Dong, ${ }^{1}$ Bing Xu, ${ }^{1}$ Bingsong Wang, ${ }^{1}$ Liqun Chu, ${ }^{1}$ and Xin Tang ${ }^{2}$ \\ ${ }^{1}$ Department of Ophthalmology, Beijing Shijitan Hospital, Capital Medical University, Beijing 100038, China \\ ${ }^{2}$ Clinical College of Ophthalmology, Tianjin Medical University, Tianjin Eye Hospital, Tianjin 300020, China \\ Correspondence should be addressed to Xin Tang; eye_tangxin@163.com
}

Received 22 June 2014; Revised 3 October 2014; Accepted 27 October 2014

Academic Editor: Denise McDonald

Copyright (C) 2015 Ning Dong et al. This is an open access article distributed under the Creative Commons Attribution License, which permits unrestricted use, distribution, and reproduction in any medium, provided the original work is properly cited.

\begin{abstract}
This study aims to ascertain whether cytokines in the aqueous humor can predict macular edema (ME) in diabetic patients following uncomplicated phacoemulsification cataract surgery. Undiluted aqueous humor samples were obtained from 136 consecutive type 2 diabetic patients who underwent cataract surgery. The concentrations of 27 cytokines were measured in aqueous humor using the multiplex bead immunoassay. At the final follow-up examination, 116 patients completed 4 weeks of follow-up, and the incidence of macular edema was $29.31 \%$ (34 patients) 4 weeks after cataract surgery. Compared to the ME ( - ) patients, the concentrations of interleukin-1 $\beta$ (IL-1 $\beta)(P<0.001)$, IL-6 $(P<0.001)$, IL-8 $(P<0.001)$, interferon-induced protein-10 (IP-10) $(P=0.003)$, monocyte chemotactic protein-1 (MCP-1) $(P<0.001)$, and vascular endothelial growth factor (VEGF) $(P<0.001)$ in the ME $(+)$ patients were significantly higher. In addition, the aqueous levels of IL-1 $\beta(r=0.288)$, IL-6 $(r=0.345)$, IL-8 $(r=0.256)$, IP-10 $(r=0.377)$, MCP-1 $(r=0.423)$, and VEGF $(r=0.279)$ were positively correlated with the postoperative foveal center point thickness (FCPT). However, the aqueous levels of IL-10 $(P=0.003)$ and IL-12 $(P=0.017)$ were significantly lower in patients with ME. These results suggest IL-1 $\beta$, IL-6, IL-8, IL-10, IL-12, IP-10, MCP-1, and VEGF may be potential predictors of postoperative macular thickness in patients with diabetes following uncomplicated phacoemulsification cataract surgery.
\end{abstract}

\section{Introduction}

Diabetes mellitus (DM) has been a leading public health problem in China for the last 10 years and imposes a heavy economic burden on Chinese patients [1]. Diabetic patients have been reported to have a higher prevalence of cataracts and an increased risk of developing cataracts earlier than patients without diabetes [2]. At present, the incidence of postoperative complications is decreasing with the development of phacoemulsification cataract surgery and posterior chamber intraocular lens implantation. However, anterior segment inflammation, progression of diabetic retinopathy, and macular edema (ME) are the most common complications in patients with diabetes following uncomplicated phacoemulsification cataract surgery $[3,4]$. ME is one of the main causes of unfavorable visual outcomes following uncomplicated cataract surgery and can result in permanent visual loss [5-7]. The reported incidence of ME ranges from $20 \%$ to $50 \%$ in patients with diabetes following uncomplicated phacoemulsification cataract surgery $[8,9]$.

Although the pathogenesis of macular edema is likely multifactorial and remains unknown, it appears to be associated with postoperative inflammation induced by prostaglandins or other inflammatory mediators [10, 11]. Inflammatory mediators break down the blood-retinal barrier $(\mathrm{BRB})$ and the blood-aqueous barrier (BAB), leading to increased vascular permeability [12]. A previous study measured the concentrations of VEGF and IL-6 in aqueous humor in patients with nonproliferative diabetic retinopathy by enzyme linked immunosorbent assay (ELISA) during cataract surgery [13] and demonstrated that high VEGF levels in the aqueous humor predict a significant risk of postoperative exacerbation of macular edema [13]. However, the limitations of the previous study on aqueous humor 
cytokines include the examination of a limited number of cytokines. Exploring a greater number of cytokines would provide broader insight into the inflammatory mechanisms involved. Recently, multiplex bead immunoassay has been used to detect cytokines in tears and in the aqueous humor because of the capacity of this assay to simultaneously quantify multiple cytokines in very small sample volumes [14-16].

Our previous study compared the changes in the levels of 27 aqueous humor cytokines between nondiabetic controls and type 2 diabetic patients and showed that the variety of cytokines associated with inflammation and angiogenesis may contribute to the pathogenesis of diabetic retinopathy (DR) [16]. These study participants that consisted of a consecutive cohort of diabetic patients with varying levels of retinopathy, including the absence of retinopathy, were included in this study.

Therefore, in this study, we used the multiplex bead immunoassay to evaluate the concentrations of 27 cytokines in the aqueous humor at the beginning of cataract surgery and correlate their expression levels to the development of macular edema 4 weeks after surgery. In addition, our study explores whether cytokine concentrations in the aqueous humor can predict macular edema in patients with diabetes following uncomplicated phacoemulsification cataract surgery.

\section{Materials and Methods}

2.1. Subjects. Undiluted aqueous humor samples were obtained from 136 consecutive type 2 diabetic patients (136 eyes; 71 males and 65 females) who were undergoing cataract surgery from January 2010 to April 2012. This study was approved by the Ethics Committee of Beijing Shijitan Hospital, Capital Medical University, Beijing, China, and was performed in accordance with the Declaration of Helsinki. Informed consent was obtained from all patients prior to their participation in the study.

DM was diagnosed according to the 1999 World Health Organization (WHO) criteria. Subjects were considered to have hypertension if their blood pressure was above $140 / 90 \mathrm{mmHg}$ or they were taking any antihypertensive medications. Hypercholesterolemia was defined as their fasting total plasma cholesterol was above $200 \mathrm{mg} / \mathrm{dL}$. Hypertriglyceridemia was classically defined as fasting plasma triacylglycerols (triglycerides, TG) above $200 \mathrm{mg} / \mathrm{dL}$. Inclusion criteria were the presence of diabetes mellitus and the absence of any retinal or optic nerve disease except diabetic retinopathy in the study group. The exclusion criteria included (1) any other ocular condition (e.g., glaucoma, uveitis), (2) a history of ocular surgery, (3) a history of ocular inflammation, and (4) current presence or history of clinically significant macular edema (CSME).

2.2. Procedure. Patients underwent preoperative ophthalmologic examination and a physical examination that included best-corrected visual acuity (BCVA), slit lampassisted biomicroscopy of the anterior segment, a fundus examination, and optical coherence tomography (OCT), which was used to measure the foveal center point thickness (FCPT). The BCVA was measured with a Snellen chart at the preoperative examination, 1 day and 4 weeks postoperatively. The OCT examination (Stratus OCT3; Carl Zeiss Meditec, Dublin, California, USA) was performed by an experienced operator through a dilated pupil. Each study eye underwent OCT testing fewer than 2 weeks before cataract surgery and 4 weeks postoperatively. OCT images were generated with the use of six radial-line scans, $6.00 \mathrm{~mm}$ each in length. The maximal foveal center point thickness (in micrometers) was measured at the center point of the fovea by manually placing computerized calipers at the vitreous-retina and retina-retinal pigment epithelium interfaces $[5,17]$.

2.3. Surgical Technique. All cataract surgeries were performed using the phacoemulsification technique and the insertion of a foldable hydrophilic acrylic intraocular lens (AcrySof IQ IOL, Alcon, Inc.) in the capsular bag. A total of $0.3 \mathrm{mg}$ TobraDex ointment (tobramycin $0.3 \%$ and dexamethasone $0.1 \%$, Alcon, Inc.) was used at the end of the surgery in all patients. All patients were instructed to administer TobraDex eye drops (tobramycin $0.3 \%$ and dexamethasone $0.1 \%$, Alcon, Inc.) 4 times daily for 2 weeks after surgery and 2 times daily until 4 weeks after cataract surgery. In addition, all patients were instructed to administer $0.1 \%$ Diclofenac sodium eye drops 4 times daily for 4 weeks after surgery. All patients were followed for at least 4 weeks after surgery.

2.4. Aqueous Humor Sampling. At the time of cataract surgery, a sterile lid speculum was placed, and a sterile tuberculin syringe was placed in the temporal limbal quadrant. Once inserted, undiluted aqueous humor samples (0.1-0.2 mL) were aspirated into a syringe. The samples were immediately frozen and stored at $-80^{\circ} \mathrm{C}$ until analysis.

2.5. Postoperative Evaluation. Postoperative follow-up visits were scheduled for 1 day and 4 weeks after cataract surgery. The following assessments were performed 1 day after cataract surgery: BCVA, slit lamp-assisted biomicroscopy, fundus examination, and IOP. The following assessments were performed 4 weeks after cataract surgery: BCVA, slit lampassisted biomicroscopy, IOP, fundus examination, and OCT.

2.6. Definition of Postoperative Macular Edema. Macular edema was defined as an increase in the center point thickness of more than $30 \%$ from preoperative baseline on OCT 4 weeks after cataract surgery $[5,17]$. All patients were divided into either the macular edema group $[\mathrm{ME}(+)]$ or nonmacular edema group $[\mathrm{ME}(-)]$.

2.7. Multiplex Analysis of Cytokines in Aqueous Humors. The Bio-Plex ProTM magnetic color bead-based multiplex assay (Bio-Plex Human Cytokine 27-plex panel; Bio-Rad, Hercules, CA) was used to measure the concentrations of twenty-seven human aqueous humor cytokines: interleukin$1 \beta$ (IL-1 $\beta)$, IL-1r $\alpha$, IL-2, IL-4, IL-5, IL-6, IL-7, IL-8, IL-9, IL-10, IL-12, IL-13, IL-15, IL-17, basic fibroblast growth factor (b-FGF), EOTAXIN, granulocyte colony-stimulating factor (G-CSF), granulocyte macrophage colony-stimulating 
factor (GMCSF), interferon-gamma (IFN- $\gamma$ ), interferoninduced protein-10 (IP-10 or CXCL10), monocyte chemotactic protein-1 (MCP-1 or CCL2), macrophage inflammatory protein-1 $\alpha$ (MIP-1 $\alpha$ or CCL3), macrophage inflammatory protein-1 $\beta$ (MIP- $1 \beta$ or CCL4), platelet-derived growth factorBB (PDGF-BB), regulated upon activation normal T-cell expressed and secreted (RANTES), tumor necrosis factoralpha (TNF- $\alpha$ ), and vascular endothelial growth factor (VEGF). The analysis procedure was performed according to the manufacturer's instructions. Standard curves were generated using the Bio-PlexTM 200 System (software version 6.0; Bio-Rad Laboratories) and were used to calculate the cytokine concentrations in the aqueous humor samples.

2.8. Statistical Analysis. Data were recorded as the mean \pm $\mathrm{SD}$ or as the median and range. The BCVA values were converted to logarithm of the minimum angle of resolution $(\log$ MAR). The statistical analyses were performed using SPSS for Windows Version 17.0. The Pearson $\chi^{2}$ test was used to compare the proportion of qualitative variables. The Student's $t$-test and Mann-Whitney $U$ test were used to compare means of quantitative variables between two independent groups. The Kruskal-Wallis test was used to compare multiple groups. Pearson correlation coefficients were used to assess the relationship between the concentrations of assayed cytokines and the foveal center point thickness 4 weeks after cataract surgery. A $P$ value less than 0.05 was accepted as statistically significant.

\section{Results}

3.1. Patient Demographics. A total of 136 consecutive type 2 diabetic patients (136 eyes; 71 males and 65 females) were enrolled, and there were no cases of intraoperative vitreous loss or suprachoroidal hemorrhage. Twelve patients were excluded for currently present macular edema. At the final follow-up examination, 116 patients (116 eyes) completed 4 weeks of follow-up ( $93.5 \%$ completion), and the eight patients who did not complete the protocol were excluded from the study. In the entire study population, 24 eyes (20.7\%) were nondiabetic retinopathy, 45 eyes $(38.8 \%)$ were mild nonproliferative diabetic retinopathy, 31 eyes (26.7\%) were moderate nonproliferative diabetic retinopathy, 8 eyes (6.9\%) were severe nonproliferative diabetic retinopathy, and 8 eyes (6.9\%) were proliferative diabetic retinopathy. At the final follow-up examination, 34 patients (34 eyes; 16 males and 18 females) had an increase in their center point thickness of more than $30 \%$ from the preoperative baseline on OCT 4 weeks after cataract surgery. The incidence of macular edema was $29.31 \%$. Table 1 shows demographic and clinical characteristics of patients, including the 34 consecutive ME (+) patients and $82 \mathrm{ME}(-)$ patients (82 eyes; 49 males and 33 females). There were no significant differences in age, hypertension, blood glucose level, cholesterol, triglycerides, type of cataract, and iris color between the $\mathrm{ME}(+)$ and $\mathrm{ME}$ $(-)$ groups.

3.2. Postoperative Clinical Characteristics. The mean BCVA before surgery was $0.61 \pm 0.19(\log M A R)$ in the $\mathrm{ME}(-)$ group
TABLE 1: Baseline characteristics of patients with ME (-) and ME $(+)$.

\begin{tabular}{lccc}
\hline Characteristics & ME (-) & ME (+) & $P$ value \\
\hline Number & 82 & 34 & - \\
Gender & & & $0.210^{\mathrm{a}}$ \\
$\quad$ Male (\%) & $49(59.7)$ & $16(47.1)$ & \\
$\quad$ Female (\%) & $33(40.3)$ & $18(52.9)$ & \\
Age (SD) & $64.8(6.33)$ & $67.6(8.06)$ & $0.310^{\mathrm{b}}$ \\
Hypertension (\%) & $51(62.2)$ & $19(55.9)$ & $0.527^{\mathrm{a}}$ \\
Hypercholesterolemia (\%) & $20(24.4)$ & $13(38.2)$ & $0.132^{\mathrm{a}}$ \\
Hypertriglyceridemia (\%) & $22(26.8)$ & $11(32.4)$ & $0.548^{\mathrm{a}}$ \\
Blood glucose level, mmol/L (SD) & $7.8(2.15)$ & $8.6(2.56)$ & $0.123^{\mathrm{b}}$ \\
Glycosylated hemoglobin (SD) & $7.5(2.23)$ & $8.03(1.85)$ & $0.225^{\mathrm{c}}$ \\
Type of cataract & & & $0.205^{\mathrm{a}}$ \\
$\quad$ Cortical (\%) & $28(34.2)$ & $6(17.7)$ & \\
$\quad$ Nuclear (\%) & $38(46.3)$ & $20(58.8)$ & \\
Posterior subcapsular (\%) & $16(19.5)$ & $8(23.5)$ & \\
Iris colour & & & $0.480^{\mathrm{a}}$ \\
$\quad$ Dark (\%) & $60(73.2)$ & $27(79.4)$ & \\
$\quad$ Light (\%) & $22(26.8)$ & $7(20.6)$ & \\
\hline
\end{tabular}

${ }^{\mathrm{a}}$ Pearson $\chi^{2}$ test; ${ }^{\mathrm{b}}$ Student's $t$-test; ${ }^{\mathrm{c}}$ Mann-Whitney $U$ test.

TABLE 2: Preoperative, 1-day, and 4-week postcataract surgery BCVA for eyes with ME (-) and ME (+).

\begin{tabular}{lccc}
\hline & \multicolumn{2}{c}{$\log$ MAR BCVA } & \multirow{2}{*}{$P$ value $^{\mathrm{a}}$} \\
& $\mathrm{ME}(-), n=82$ & $\mathrm{ME}(+), n=34$ & \\
\hline Preoperative & $0.61 \pm 0.19$ & $0.65 \pm 0.20$ & 0.275 \\
1-day & $0.19 \pm 0.13$ & $0.22 \pm 0.16$ & 0.341 \\
4-week & $0.14 \pm 0.12$ & $0.29 \pm 0.15$ & $<0.001$ \\
\hline
\end{tabular}

log MAR: logarithm of the minimum angle of resolution; BCVA: bestcorrected visual acuity.

${ }^{\mathrm{a}}$ Student's $t$-test.

TABLE 3: Preoperative and 4-week postcataract surgery foveal center point thickness for eyes with ME (-) and ME (+).

\begin{tabular}{lccc}
\hline & \multicolumn{3}{c}{ Foveal center point thickness (mean \pm SD; $\mu \mathrm{m})$} \\
& $\mathrm{ME}(-), n=82$ & $\mathrm{ME}(+), n=34$ & $P$ value $^{\mathrm{a}}$ \\
\hline Preoperative & $159.93 \pm 19.84$ & $162.41 \pm 21.33$ & 0.091 \\
4-week & $185.53 \pm 18.35$ & $237.24 \pm 24.16$ & $<0.001$ \\
$P$ value $^{\mathrm{a}}$ & 0.118 & 0.003 & \\
\hline
\end{tabular}

${ }^{\mathrm{a}}$ Student's $t$-test.

and $0.65 \pm 0.20(\log \mathrm{MAR})$ in the ME $(+)$ group. Table 2 shows the BCVA 1 day and 4 weeks after surgery. The postoperative BCVA was not significantly different between the ME $(-)$ and $\mathrm{ME}(+)$ groups 1 day after surgery. However, the mean BCVA 4 weeks after surgery was improved compared to the mean BCVA 1 day after surgery in the ME (-) group. Conversely, the mean BCVA 4 weeks after surgery was decreased compared to the mean BCVA 1 day after surgery in the ME (+) group. In addition, the postoperative BCVA was significantly different between the ME (-) and ME (+) groups 4 weeks after surgery $(P<0.001)$. 
TABLE 4: The concentrations of cytokines in aqueous humors of eyes with ME (-) and ME (+) (pg/mL).

\begin{tabular}{|c|c|c|c|c|c|}
\hline \multirow{2}{*}{ Cytokine } & \multicolumn{2}{|c|}{$\operatorname{ME}(-), n=82$} & \multicolumn{2}{|c|}{$\mathrm{ME}(+), n=34$} & \multirow{2}{*}{$P$ value $^{\mathrm{a}}$} \\
\hline & Median & Range & Median & Range & \\
\hline IL-1 $\beta$ & 4.2 & $0-76$ & 8.6 & $0-102$ & $<0.001$ \\
\hline IL-1r $\alpha$ & 13.2 & $0-325$ & 18.1 & $0-336$ & 0.445 \\
\hline IL-2 & 1.5 & $0-96$ & 1.7 & $0-106$ & 0.578 \\
\hline IL-4 & 1.2 & $0-105$ & 1.5 & $0-124$ & 0.862 \\
\hline IL-5 & 1.1 & $0-133$ & 1.3 & $0-126$ & 0.653 \\
\hline IL-6 & 19.8 & $0-226$ & 28.5 & $0-362$ & $<0.001$ \\
\hline IL-7 & 4.5 & $0-82$ & 2.7 & $0-86$ & 0.203 \\
\hline IL-8 & 12.6 & $0-123$ & 17.3 & $0-186$ & $<0.001$ \\
\hline IL-9 & 3.1 & $0-102$ & 3.3 & $0-169$ & 0.580 \\
\hline IL-10 & 8.2 & $0-23$ & 5.6 & $0-21$ & 0.003 \\
\hline IL-12 & 7.2 & $0-42$ & 4.6 & $0-36$ & 0.017 \\
\hline IL-13 & 2.1 & $0-26$ & 1.9 & $0-36$ & 0.453 \\
\hline IL-15 & 1.6 & $0-56$ & 1.8 & $0-38$ & 0.686 \\
\hline IL-17 & - & - & - & - & - \\
\hline b-FGF & 12.4 & $0-165$ & 11.3 & $0-156$ & 0.560 \\
\hline Eotaxin & 5.9 & $0-86$ & 6.2 & $0-95$ & 0.753 \\
\hline G-CSF & - & - & - & - & - \\
\hline GM-CSF & 9.2 & $0-86$ & 9.8 & $0-79$ & 0.876 \\
\hline IFN- $\gamma$ & - & - & - & - & - \\
\hline IP-10 & 3.3 & $0-56$ & 5.1 & $0-72$ & 0.003 \\
\hline MCP-1 & 189.5 & $58-1623$ & 325.6 & $124-2388$ & $<0.001$ \\
\hline MIP-1 $1 \alpha$ & - & - & - & - & - \\
\hline MIP-1 $\beta$ & 27.8 & $0-156$ & 26.5 & $0-178$ & 0.539 \\
\hline PDGF-BB & 3.3 & $0-45$ & 3.1 & $0-42$ & 0.756 \\
\hline RANTES & 4.6 & $0-75$ & 4.9 & $0-76$ & 0.577 \\
\hline TNF- $\alpha$ & - & - & - & - & - \\
\hline VEGF & 535 & 26-1298 & 856 & $123-1756$ & $<0.001$ \\
\hline
\end{tabular}

aann-Whitney $U$ test.

The mean foveal center point thickness before surgery was $159.93 \pm 19.84 \mu \mathrm{m}$ in the ME (-) group and $162.41 \pm$ $21.33 \mu \mathrm{m}$ in the ME (+) group. Table 3 shows the FCPT 4 weeks after surgery. At 4 weeks, there was an increase of $25.6 \mu \mathrm{m}$ and $74.83 \mu \mathrm{m}$ in the FCPT of the ME (-) and ME (+) groups, respectively. The postoperative FCPT was significantly different between the ME $(-)$ and $\mathrm{ME}(+)$ groups $(P<0.001)$.

Tables 2 and 3 show that the average increase in center point thickness at 4 weeks for eyes with ME was $74.83 \mu \mathrm{m}$, which resulted in a nearly 1 -line loss of vision $(0.07$ logMAR units) compared to eyes without ME, which improved approximately 1 line of vision ( $0.05 \log$ MAR units).

3.3. Cytokines Concentrations in the Aqueous Humor. Table 4 shows the concentrations of the assayed cytokines. The positive detection rates were more than $80 \%$ for 22 cytokines. The positive detection rates for the other 5 cytokines were as follows: TNF- $\alpha$ (60\%), IL-17 (40\%), G-CSF (32\%), IFN- $\gamma(22 \%)$, and MIP- $1 \alpha(20 \%)$. These 5 cytokines were not included in the statistical analysis because of the low detection rates.
Compared to the ME (-) group, the concentrations of IL$1 \beta(P<0.001)$, IL-6 $(P<0.001)$, IL-8 $(P<0.001)$, IP-10 $(P=0.003)$, MCP-1 $(P<0.001)$, and VEGF $(P<0.001)$ from the ME (+) patients were significantly higher. However, the concentrations of IL-10 $(P=0.003)$ and IL-12 $(P=0.017)$ in the samples from the $\mathrm{ME}(+)$ patients were significantly lower than the concentrations in the ME (-) patients. There were no significant differences in other cytokine concentrations between the ME (-) and $\mathrm{ME}(+)$ patients.

\subsection{Association between Cytokines Concentrations and the} Severity of $D R$. In the 34 patients (34 eyes) with ME (+), 3 eyes (8.8\%) were nondiabetic retinopathy, 9 eyes $(26.5 \%)$ were mild nonproliferative retinopathy, 13 eyes $(38.2 \%)$ were moderate nonproliferative retinopathy, 4 eyes $(11.8 \%)$ were severe nonproliferative retinopathy, and 5 eyes (14.7\%) were proliferative retinopathy.

Tables 5 and 6 show the relationship between the concentrations of the assayed cytokines and the severity of DR. The aqueous humor levels of IL-1 $\beta$, IL-6, IL-8, MCP-1, IP-10, and VEGF increased with increasing severity of DR, and this correlation was significant. In addition, the aqueous humor levels of IL-10 and IL-12 decreased with increasing severity of $\mathrm{DR}$, and this negative correlation was significant.

3.5. Association between Cytokines Concentrations and Foveal Center Point Thickness. Table 7 shows the relationship between the concentrations of assayed cytokines and the postoperative FCPT. The aqueous levels of IL-1 $\beta(r=0.288)$, IL-6 $(r=0.345)$, IL-8 $(r=0.256)$, IP-10 $(r=0.377)$, MCP-1 $(r=0.423)$, and VEGF $(r=0.279)$ were found to positively correlate with postoperative FCPT. In addition, the aqueous level of IL-10 $(r=-0.327)$ and IL-12 $(r=-0.264)$ was negatively correlated with postoperative FCPT.

\section{Discussion}

The incidence of ME peaks at approximately 4 to 6 weeks after uneventful cataract surgery $[18,19]$. The incidence of ME has been reported to range from $4 \%$ [10] to $11 \%$ [17] in nondiabetic patients following uncomplicated phacoemulsification; however, the prevalence of ME ranges from $20 \%$ to $50 \%$ in patients with diabetes following uncomplicated phacoemulsification $[8,9]$. The different rates may be caused by the inflammation which is an important factor that induces DRrelated changes. Recent clinical and laboratory investigations have shown that diabetic subjects have an overall increased level of inflammatory activity relative to nondiabetic subjects [20-25]. Our previous study demonstrated that the levels of multiple cytokines associated with inflammation and angiogenesis in the aqueous humor from diabetic patients were increased compared to nondiabetic controls [16]. Therefore, the analysis of the aqueous humor provides useful tool in understanding the pathophysiology and treatment responses of macular edema in patients with diabetes following uncomplicated phacoemulsification cataract surgery. 
TABLE 5: Relationship between the concentrations of the assayed cytokines and the severity of DR ( $\mathrm{pg} / \mathrm{mL})$.

\begin{tabular}{lccccccc}
\hline Level $^{\mathrm{a}}$ & $N$ & IL-1 $\beta(\mathrm{SD})$ & IL-6 (SD) & IL-8 (SD) & IP-10 (SD) & MCP-1 (SD) & VEGF (SD) \\
\hline 1 & 3 & $5.1(8.4)$ & $28.4(23.1)$ & $17.3(13.3)$ & $1.8(1.5)$ & $236.7(212.8)$ & $468.6(304.3)$ \\
2 & 9 & $9.8(7.6)$ & $34.8(25.3)$ & $21.5(15.5)$ & $3.2(2.1)$ & $486.7(163.1)$ & $632.1(368.1)$ \\
3 & 13 & $13.3(11.1)$ & $42.3(31.8)$ & $25.3(13.1)$ & $7.3(3.6)$ & $656.1(256.7)$ & $787.3(357.3)$ \\
4 & 4 & $16.2(13.3)$ & $76.2(47.3)$ & $35.8(29.7)$ & $12.2(9.1)$ & $837.6(367.9)$ & $867.4(423.8)$ \\
5 & 5 & $21.7(14.2)$ & $121.7(69.3)$ & $65.8(52.1)$ & $18.4(17.9)$ & $1306.5(683.4)$ & $976.2(476.4)$ \\
$P$ value $^{b}$ & & $<0.001$ & $<0.001$ & $<0.001$ & $<0.001$ & $<0.001$ & $<0.001$ \\
\hline
\end{tabular}

${ }^{a}$ Level: 1 = nondiabetic retinopathy; 2 = mild nonproliferative retinopathy; 3 = moderate nonproliferative retinopathy; 4 = severe nonproliferative retinopathy; $5=$ proliferative retinopathy.

${ }^{\mathrm{b}}$ Kruskal-Wallis test.

TABLE 6: Relationship between the concentrations of the assayed cytokines and the severity of DR $(\mathrm{pg} / \mathrm{mL})$.

\begin{tabular}{lccc}
\hline Level $^{\mathrm{a}}$ & $N$ & IL-10 (SD) & IL-12 (SD) \\
\hline 1 & 3 & $9.8(4.3)$ & $17.1(9.3)$ \\
2 & 9 & $7.2(3.5)$ & $14.6(7.6)$ \\
3 & 13 & $5.6(4.6)$ & $12.3(8.5)$ \\
4 & 4 & $4.9(3.8)$ & $9.2(7.7)$ \\
5 & 5 & $4.6(3.2)$ & $7.1(6.2)$ \\
$P$ value $^{\mathrm{b}}$ & & $<0.001$ & $<0.001$ \\
\hline
\end{tabular}

${ }^{a}$ Level: 1 = nondiabetic retinopathy; 2 = mild nonproliferative retinopathy; 3 = moderate nonproliferative retinopathy; 4 = severe nonproliferative retinopathy; 5 = proliferative retinopathy.

${ }^{\mathrm{b}}$ Kruskal-Wallis test.

TABLE 7: Correlations between concentrations cytokines in aqueous humors and 4-week postcataract surgery foveal center point thickness.

\begin{tabular}{lcc}
\hline Cytokine & Correlation coefficients & $P$ value $^{\mathrm{a}}$ \\
\hline IL-1 $\beta$ & 0.288 & 0.005 \\
IL-6 & 0.345 & 0.008 \\
IL-8 & 0.256 & 0.016 \\
IP-10 & 0.377 & 0.007 \\
MCP-1 & 0.423 & 0.001 \\
VEGF & 0.279 & 0.012 \\
IL-10 & -0.327 & 0.013 \\
IL-12 & -0.264 & 0.036 \\
\hline
\end{tabular}

${ }^{\mathrm{a}}$ Pearson correlation coefficient.

Consistent with previous studies $[8,9]$, our study showed that 34 patients (34 eyes; 16 males and 18 females) had an increase in their center point thickness of more than $30 \%$ from the preoperative baseline on OCT 4 weeks after cataract surgery. The incidence of macular edema was $29.31 \%$. The average increase in center point thickness at 4 weeks for eyes with ME was $74.83 \mu \mathrm{m}$, which resulted in a nearly 1line loss of vision (0.07 logMAR units) compared to eyes without ME, which improved approximately 1 line of vision (0.05 logMAR units). All these show that ME is a main cause of poor postoperative visual gain following uncomplicated cataract surgery.

The pathophysiology of ME involves the accumulation of transudate in the outer plexiform and inner nuclear layers of the retina; the microcysts coalesce into cysts [26]. The pathogenesis of $\mathrm{ME}$ is associated with the destruction of BRB and $\mathrm{BAB}$ induced by prostaglandins or other inflammatory mediators [10-12]. Elevated levels of angiogenic factors, inflammatory cytokines, chemokines, and growth factors in the aqueous humor may play a role in the breakdown of the vascular barrier $[22,23,27,28]$.

Aqueous analysis provides useful tools in understanding the pathophysiology and treatment response to many ocular conditions. However, aqueous samples consist of very small volumes, limiting the usefulness of the analysis with traditional ELISA techniques. In the current study, multiplex bead immunoassay was used to analyze the aqueous humor levels of cytokines and chemokines in diabetic patients following uncomplicated phacoemulsification cataract surgery. To our knowledge, this is a comparatively large number of samples, and it is the first investigation of these 27 aqueous cytokines as predictors of macular edema in diabetic patients following uncomplicated phacoemulsification cataract surgery.

In our study, positive detection rates were more than $80 \%$ for 22 cytokines. Compared to the ME (-) group, the concentrations of IL-1 $\beta(P<0.001)$, IL-6 $(P<0.001)$, IL$8(P<0.001)$, IP-10 $(P=0.003), \operatorname{MCP}-1(P<0.001)$, and $\operatorname{VEGF}(P<0.001)$ from the $\mathrm{ME}(+)$ patients were significantly higher. However, the concentrations of IL-10 $(P=0.003)$ and IL-12 $(P=0.017)$ in the samples from the ME $(+)$ patients were significantly lower than the concentrations in the ME $(-)$ patients.

IL- $1 \beta$ is a proinflammatory cytokine and an angiogenic mediator in several systems in diabetic patients, including the aqueous humor, vitreous, and tears $[29,30]$. IL-1 $\beta$ upregulates several inflammatory mediators, including IL$1 \beta$ itself, TNF- $\alpha$, cyclooxygenase 2 (COX-2), prostaglandins, inducible nitric oxide synthase (iNOS), and chemokines [31]. The intravitreal injection of IL- $1 \beta$ accelerates the apoptosis of retinal capillary cells via the activation of $\mathrm{NF}-\kappa \mathrm{B}$, and this process is exacerbated under high-glucose conditions [32]. A previous study demonstrated that animals were protected from diabetes-induced retinal pathology by IL- $1 \beta$ receptor knock-out [33]. In the current study, the IL- $1 \beta$ concentrations in the $\mathrm{ME}(+)$ patients were significantly higher than those of the ME (-) group. Our study suggests a possible role of IL-1 $\beta$ in the development of ME after cataract surgery.

It is well known that IL-6 is a multifunctional cytokine that has proinflammatory and angiogenic functions through 
the induction of VEGF [22]. In addition, it has been reported that IL- 6 is involved in the breakdown of the blood-retinal barrier [34]. In the patients with DR, the level of inflammation gradually increases as the proliferative pathogenic process and neovascularization progress. In our study, the concentrations of IL- 6 from the ME (+) patients were significantly higher than those of the ME (-) patients. There is evidence that inflammation is an important molecular mechanism in the development and progression of ME after uncomplicated phacoemulsification cataract surgery.

Chemokines, including four subfamilies, C, CC, CXC, and $\mathrm{CX} 3 \mathrm{C}$, are small heparin-binding proteins that bind to their cognate G-protein coupled receptors (GPCRs) to elicit cellular responses [35, 36]. IL- 8 and IP-10 are categorized as CXC chemokines, and MCP-1 is categorized as a CC chemokine. IL- 8 is the major attractant and activator of neutrophils and $\mathrm{T}$ lymphocytes but not monocytes, and increased levels of IL-8 in PDR are associated with a higher extent of large-vessel gliotic obliteration [35]. IP-10 selectively attracts activated $\mathrm{T}$ lymphocytes, which are the only inflammatory cells that express the chemokine receptor CXCR3 [36]. MCP-1 regulates the migration and infiltration of monocytes/macrophages via the chemokine receptor CCR2 but has no effect on neutrophils [37]. In our study, the concentrations of IL-8, IP-10 and MCP-1 from the ME (+) patients were significantly higher than those of the ME (-) patients. All of the evidence indicates that inflammation is an important molecular mechanism in the development and progression of ME after uncomplicated phacoemulsification cataract surgery.

Vascular endothelial growth factor (VEGF) is an endothelial cell mitogen that induces increases in vascular permeability and angiogenesis, enhances collateral vessel formation, and increases the permeability of the microvasculature [38]. The aqueous humor levels of VEGF have been found to be markedly increased in patients with DR, and the VEGF level has been reported to be significantly correlated with the severity of diabetic retinopathy [39]. In accordance with previous results, levels of VEGF in the aqueous have been found to be markedly increased in postoperative exacerbation of macular edema patients [13]; therefore, aqueous cytokine may be a predictor of macular edema in diabetic patients after cataract surgery.

IL-10, which is produced by monocytes and macrophages, is one of the main anti-inflammatory cytokines. IL-10 limits inflammation by reducing the synthesis of proinflammatory cytokines such as IL-1 and TNF- $\alpha$, by suppressing cytokine receptor expression and by inhibiting receptor activation [40]. In addition, IL-10 prevents angiogenesis by downregulating VEGF expression [41]. IL-12 possesses antiangiogenic activity that is mediated by the stimulation of T-helper lymphocytes and the induction of IP-10 expression [42]. In our study, the IL-10 and IL-12 concentrations of samples from the $\mathrm{ME}(+)$ patients were significantly lower. Our results suggest that low levels of circulating IL-10 (anti-inflammatory and antiangiogenic activity) and IL-12 (antiangiogenic) are involved in the pathogenesis of ME after cataract surgery.

The limitations of our study should be noted. First, the number of patients with severe PDR enrolled in the study was relatively low. Aqueous cytokines as predictors of macular edema in patients with severe PDR following uncomplicated cataract surgery needs to be studied further. Second, the concentrations of the cytokines in vitreous samples and serum were not determined. The cytokine levels in the vitreous are usually higher, and the analysis of vitreous would more accurately reflect the intraocular levels of cytokines and the status of the retina. However, in contrast to vitreous samples, obtaining aqueous fluid samples from the anterior chamber is easier, faster, and less risky. In addition, the ME was assessed at 4 weeks of cataract surgery and consequently the ME formation may also be the result of surgery related anterior segment inflammation during this period. Hence, in order to reduce the influence of operation on the result of $\mathrm{ME}$, all phacoemulsification cataract extractions were performed by the same surgeon. Finally, multiplex bead immunoassay has the limitation if the cytokine levels are very low, so the positive detection rates for the 5 cytokines were not more than $80 \%$ and these cytokines were not included in the statistical analysis because of the low detection rates in the current study.

\section{Conclusions}

The present study showed that aqueous levels of IL- $1 \beta$, IL-6, IL-8, IP-10, MCP-1, and VEGF were increased in patients with postcataract surgery macular edema and were positively correlated with FCPT 4 weeks following cataract surgery in diabetic patients. In addition, aqueous levels of IL-10 and IL-12 were significantly lower in patients with postcataract surgery ME and were negatively correlated with postoperative FCPT. These results indicate that IL- $1 \beta$, IL-6, IL-8, IL-10, IL-12, IP-10, MCP-1, and VEGF may be potential predictors to determine postoperative macular thickness in diabetic patients following uncomplicated phacoemulsification cataract surgery.

\section{Disclosure}

This work neither has been published nor is it being considered for publication elsewhere in any form. All authors have read and approved the paper.

\section{Conflict of Interests}

The authors declare that there is no conflict of interests regarding the publication of this paper.

\section{Acknowledgment}

This work is supported by research grants from National Natural Science Foundation for the Young Scholars of Beijing Shijitan Hospital, Capital Medical University (no. 2013QB01).

\section{References}

[1] W. P. Jia, C. Pang, L. Chen et al., "Epidemiological characteristics of diabetes mellitus and impaired glucose regulation in a Chinese adult population: the Shanghai Diabetes Studies, 
a cross-sectional 3-year follow-up study in Shanghai urban communities," Diabetologia, vol. 50, no. 2, pp. 286-292, 2007.

[2] B. E. K. Klein, R. Klein, and S. E. Moss, "Prevalence of cataracts in a population-based study of persons with diabetes mellitus," Ophthalmology, vol. 92, no. 9, pp. 1191-1196, 1985.

[3] M. Vukicevic, T. Gin, and S. Al-Qureshi, "Prevalence of optical coherence tomography-diagnosed postoperative cystoid macular oedema in patients following uncomplicated phacoemulsification cataract surgery," Clinical and Experimental Ophthalmology, vol. 40, no. 3, pp. 282-287, 2012.

[4] U. Menchini, S. Cappelli, and G. Virgili, "Cataract surgery and diabetic retinopathy," Seminars in Ophthalmology, vol. 18, no. 3, pp. 103-108, 2003.

[5] M. Dieleman, R. J. Wubbels, M. Van Kooten-Noordzij, and P. W. T. de Waard, "Single perioperative subconjunctival steroid depot versus postoperative steroid eyedrops to prevent intraocular inflammation and macular edema after cataract surgery," Journal of Cataract and Refractive Surgery, vol. 37, no. 9, pp. 1589-1597, 2011.

[6] A. Pollack, H. Leiba, A. Bukelman, and M. Oliver, "Cystoid macular oedema following cataract extraction in patients with diabetes," British Journal of Ophthalmology, vol. 76, no. 4, pp. 221-224, 1992.

[7] W. E. Benson, G. C. Brown, W. Tasman, J. A. McNamara, and J. F. Vander, "Extracapsular cataract extraction with placement of a posterior chamber lens in patients with diabetic retinopathy," Ophthalmology, vol. 100, no. 5, pp. 730-738, 1993.

[8] R. A. Mittra, J. L. Borrillo, S. Dev, W. F. Mieler, and S. B. Koenig, "Retinopathy progression and visual outcomes after phacoemulsification in patients with diabetes mellitus," Archives of Ophthalmology, vol. 118, no. 7, pp. 912-917, 2000.

[9] J. Chung, M. Y. Kim, H. S. Kim, J. S. Yoo, and Y. C. Lee, "Effect of cataract surgery on the progression of diabetic retinopathy," Journal of Cataract and Refractive Surgery, vol. 28, no. 4, pp. 626-630, 2002.

[10] M.-L. Bélair, S. J. Kim, J. E. Thorne et al., "Incidence of cystoid macular edema after cataract surgery in patients with and without uveitis using optical coherence tomography," American Journal of Ophthalmology, vol. 148, no. 1, pp. 128-135, 2009.

[11] G. Gulkilik, S. Kocabora, M. Taskapili, and G. Engin, "Cystoid macular edema after phacoemulsification: risk factors and effect on visual acuity," Canadian Journal of Ophthalmology, vol. 41, no. 6, pp. 699-703, 2006.

[12] N. R. Benitah and J. G. Arroyo, "Pseudophakic cystoid macular edema," International Ophthalmology Clinics, vol. 50, no. 1, pp. 139-153, 2010.

[13] H. Funatsu, H. Yamashita, H. Noma, E. Shimizu, T. Mimura, and S. Hori, "Prediction of macular edema exacerbation after phacoemulsification in patients with nonproliferative diabetic retinopathy," Journal of Cataract and Refractive Surgery, vol. 28, no. 8, pp. 1355-1363, 2002.

[14] J. Liu, B. Shi, S. He, X. Yao, M. D. P. Willcox, and Z. Zhao, "Changes to tear cytokines of type 2 diabetic patients with or without retinopathy," Molecular Vision, vol. 16, pp. 2931-2938, 2010.

[15] L. Chu, B. Wang, B. Xu, and N. Dong, "Aqueous cytokines as predictors of macular edema in non-diabetic patients following uncomplicated phacoemulsification cataract surgery," Molecular Vision, vol. 19, pp. 2418-2425, 2013.

[16] N. Dong, B. Xu, B. Wang, and L. Chu, "Study of 27 aqueous humor cytokines in patients with type 2 diabetes with or without retinopathy," Molecular Vision, vol. 19, pp. 1734-1746, 2013.

[17] S. J. Kim, R. Equi, and N. M. Bressler, "Analysis of macular edema after cataract surgery in patients with diabetes using optical coherence tomography," Ophthalmology, vol. 114, no. 5, pp. 881-889, 2007.

[18] I. Perente, C. A. Utine, C. Ozturker et al., "Evaluation of macular changes after uncomplicated phacoemulsification surgery by optical coherence tomography," Current Eye Research, vol. 32, no. 3, pp. 241-247, 2007.

[19] H. Z. Buyukyildiz, G. Gulkilik, and Y. Z. Kumcuoglu, "Early serous macular detachment after phacoemulsification surgery," Journal of Cataract and Refractive Surgery, vol. 36, no. 11, pp. 1999-2002, 2010.

[20] C. Hernández, R. M. Segura, A. Fonollosa, E. Carrasco, G. Francisco, and R. Simó, "Interleukin-8, monocyte chemoattractant protein-1 and IL-10 in the vitreous fluid of patients with proliferative diabetic retinopathy," Diabetic Medicine, vol. 22, no. 6, pp. 719-722, 2005.

[21] A. M. Abu El-Asrar, S. Struyf, D. Kangave, K. Geboes, and J. van Damme, "Chemokines in proliferative diabetic retinopathy and proliferative vitreoretinopathy," European Cytokine Network, vol. 17, no. 3, pp. 155-165, 2006.

[22] H. Funatsu, H. Yamashita, T. Ikeda, T. Mimura, S. Eguchi, and S. Hori, "Vitreous levels of interleukin-6 and vascular endothelial growth factor are related to diabetic macular edema," Ophthalmology, vol. 110, no. 9, pp. 1690-1696, 2003.

[23] B. T. Ozturk, B. Bozkurt, H. Kerimoglu, M. Okka, U. Kamis, and K. Gunduz, "Effect of serum cytokines and VEGF levels on diabetic retinopathy and macular thickness," Molecular Vision, vol. 15, pp. 1906-1914, 2009.

[24] Y. Mitamura, S. Takeuchi, A. Matsuda, Y. Tagawa, Y. Mizue, and J. Nishihira, "Monocyte chemotactic protein-1 in the vitreous of patients with proliferative diabetic retinopathy," Ophthalmologica, vol. 215, no. 6, pp. 415-418, 2001.

[25] N. Dong, X. Li, L. Xiao, W. Yu, B. Wang, and L. Chu, "Upregulation of retinal neuronal MCP-1 in the rodent model of diabetic retinopathy and its function in vitro," Investigative Ophthalmology and Visual Science, vol. 53, no. 12, pp. 7567-7575, 2012.

[26] Y. Yonekawa and I. K. Kim, "Pseudophakic cystoid macular edema," Current Opinion in Ophthalmology, vol. 23, no. 1, pp. 26-32, 2012.

[27] M. Funk, G. Schmidinger, N. Maar et al., "Angiogenic and inflammatory markers in the intraocular fluid of eyes with diabetic macular edema and influence of therapy with bevacizumab," Retina, vol. 30, no. 9, pp. 1412-1419, 2010.

[28] H. J. Sohn, D. H. Han, I. T. Kim et al., "Changes in aqueous concentrations of various cytokines after intravitreal triamcinolone versus bevacizumab for diabetic macular edema," American Journal of Ophthalmology, vol. 152, no. 4, pp. 686-694, 2011.

[29] G. A. Limb, B. C. Little, A. Meager et al., "Cytokines in proliferative vitreoretinopathy," Eye, vol. 5, part 6, pp. 686-693, 1991.

[30] P. Murugeswari, D. Shukla, A. Rajendran, R. Kim, P. Namperumalsamy, and V. Muthukkaruppan, "Proinflammatory cytokines and angiogenic and anti-angiogenic factors in vitreous of patients with proliferative diabetic retinopathy and eales' disease," Retina, vol. 28, no. 6, pp. 817-824, 2008.

[31] N. J. Rothwell and G. N. Luheshi, "Interleukin 1 in the brain: biology, pathology and therapeutic target," Trends in Neurosciences, vol. 23, no. 12, pp. 618-625, 2000. 
[32] R. A. Kowluru and S. Odenbach, "Role of interleukin-1 $\beta$ in the pathogenesis of diabetic retinopathy," British Journal of Ophthalmology, vol. 88, no. 10, pp. 1343-1347, 2004.

[33] J. A. Vincent and S. Mohr, "Inhibition of caspase-1/interleukin$1 \beta$ signaling prevents degeneration of retinal capillaries in diabetes and galactosemia," Diabetes, vol. 56, no. 1, pp. 224-230, 2007.

[34] A. P. Moriarty, D. J. Spalton, B. J. Moriarty, J. S. Shilling, T. J. Ffytche, and M. Bulsara, "Studies of the blood-aqueous barrier in diabetes mellitus," American Journal of Ophthalmology, vol. 117, no. 6, pp. 768-771, 1994.

[35] M. G. Petrovič, P. Korošec, M. Košnik, and M. Hawlina, "Vitreous levels of interleukin-8 in patients with proliferative diabetic retinopathy," American Journal of Ophthalmology, vol. 143, no. 1, pp. 175-176, 2007.

[36] M. Loetscher, B. Gerber, P. Loetscher et al., "Chemokine receptor specific for IP10 and Mig: structure, function, and expression in activate T-lymphocytes," The Journal of Experimental Medicine, vol. 184, no. 3, pp. 963-969, 1996.

[37] L. A. Penny, "Monocyte chemoattractant protein 1 in luteolysis," Reviews of Reproduction, vol. 5, no. 2, pp. 63-66, 2000.

[38] L. P. Aiello, R. L. Avery, P. G. Arrigg et al., "Vascular endothelial growth factor in ocular fluid of patients with diabetic retinopathy and other retinal disorders," The New England Journal of Medicine, vol. 331, no. 22, pp. 1480-1487, 1994.

[39] H. Funatsu, H. Yamashita, E. Shimizu, R. Kojima, and S. Hori, "Relationship between vascular endothelial growth factor and interleukin-6 in diabetic retinopathy," Retina, vol. 21, no. 5, pp. 469-477, 2001.

[40] J. M. Rubio-Perez and J. M. Morillas-Ruiz, "A review: inflammatory process in Alzheimer's disease, role of cytokines," The Scientific World Journal, vol. 2012, Article ID 756357, 15 pages, 2012.

[41] J.-S. Silvestre, Z. Mallat, M. Duriez et al., "Antiangiogenic effect of interleukin-10 in ischemia-induced angiogenesis in mice hindlimb," Circulation Research, vol. 87, no. 6, pp. 448-452, 2000.

[42] C. Sgadari, A. L. Angiolillo, and G. Tosato, "Inhibition of angiogenesis by interleukin-12 is mediated by the interferoninducible protein 10," Blood, vol. 87, no. 9, pp. 3877-3882, 1996. 


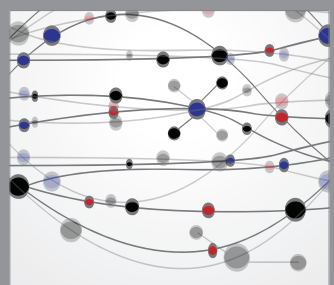

The Scientific World Journal
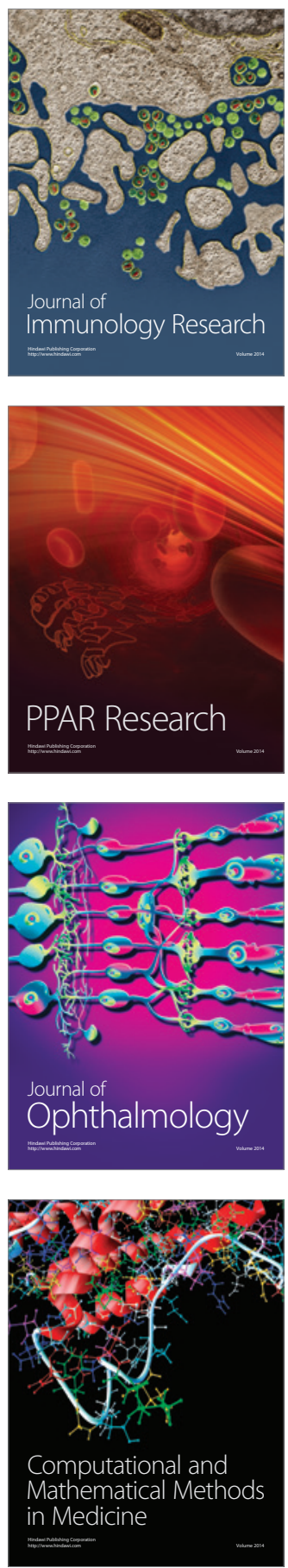

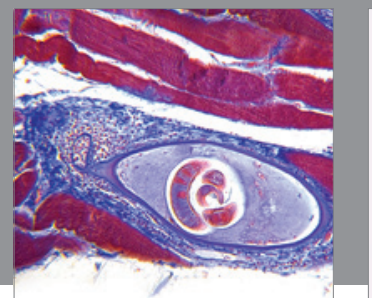

Gastroenterology

Research and Practice
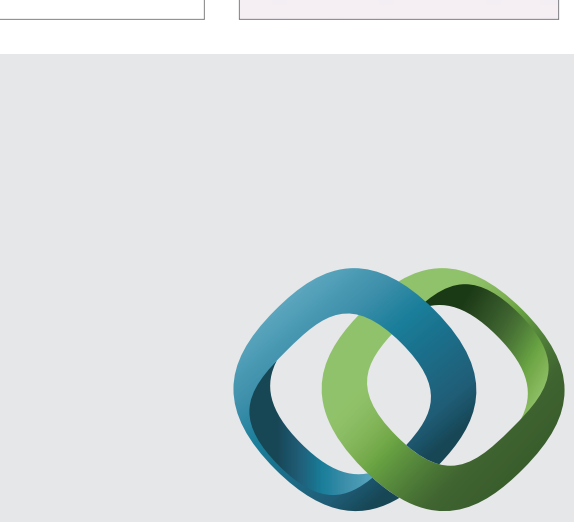

\section{Hindawi}

Submit your manuscripts at

http://www.hindawi.com
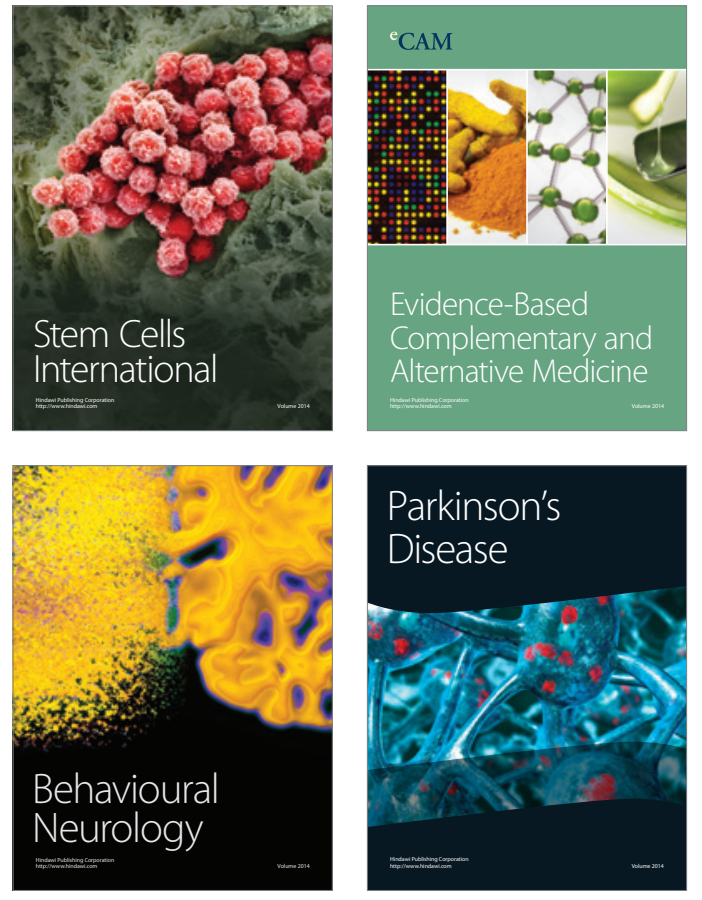
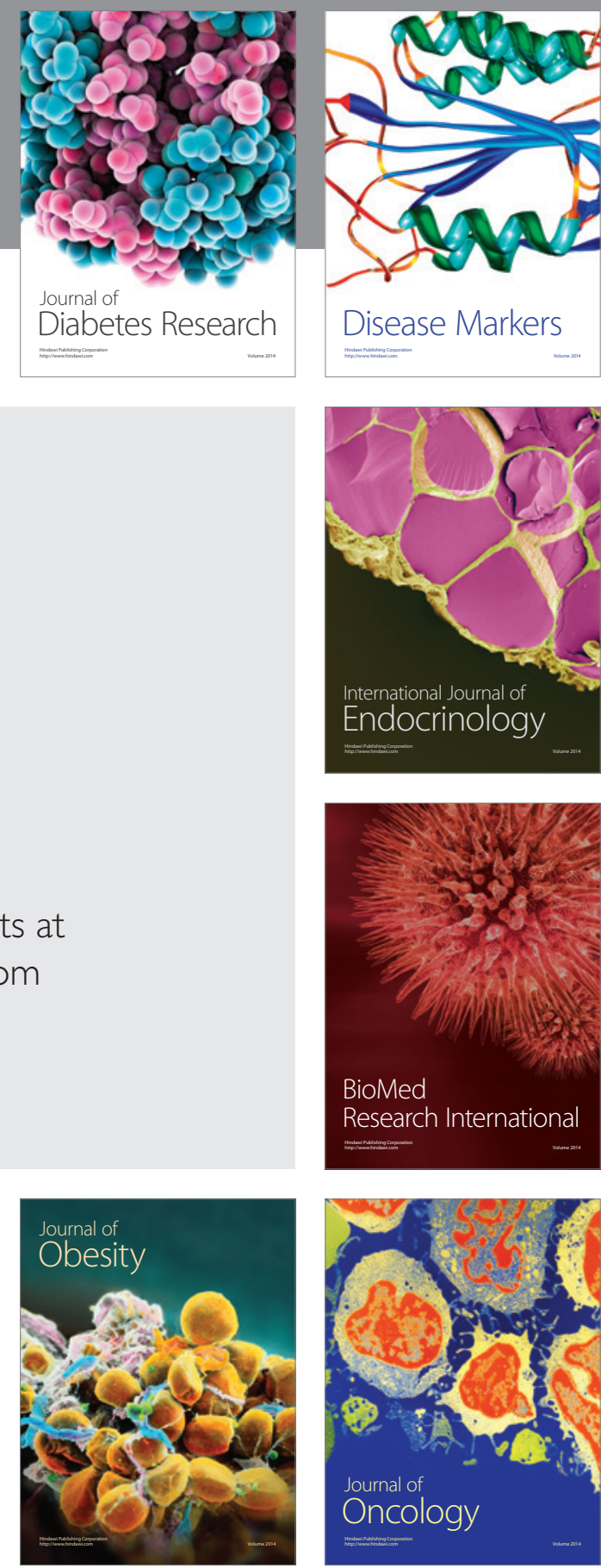

Disease Markers
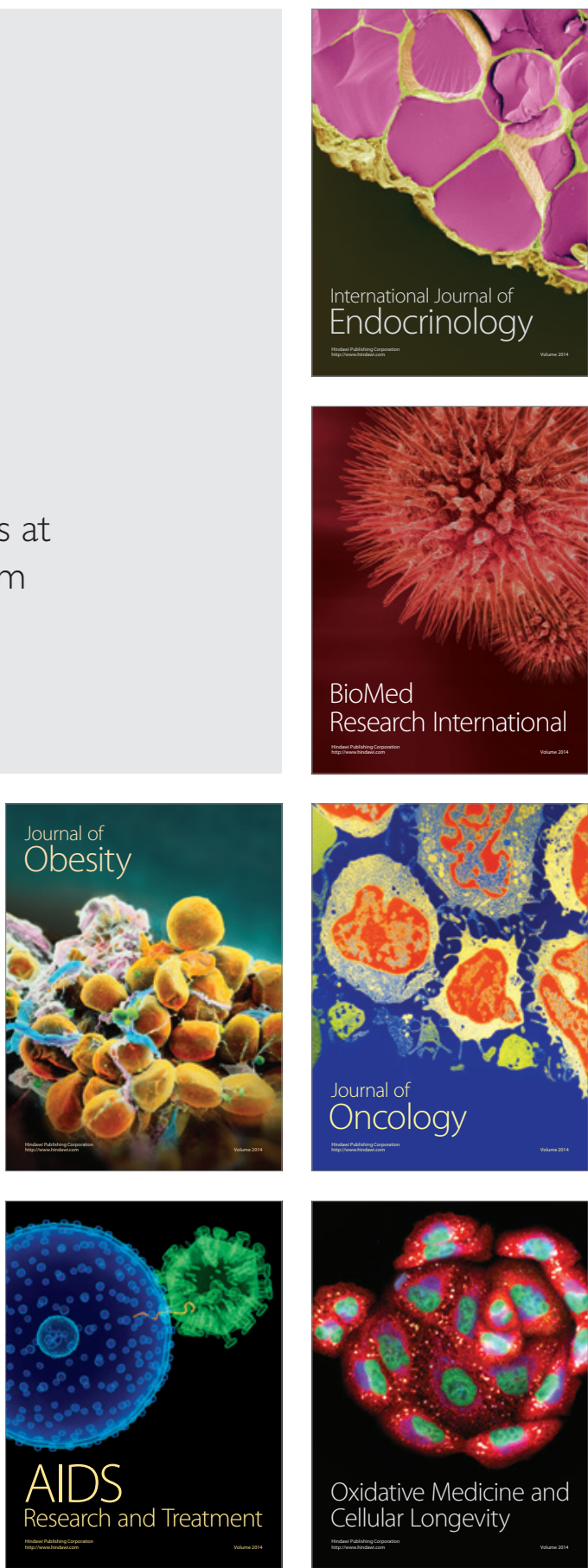